\title{
Inequality between Size and Angular Momentum for Bodies
}

\author{
Sergio Dain \\ Facultad de Matemática, Astronomía y Física, FaMAF, Universidad Nacional de Córdoba, \\ Instituto de Física Enrique Gaviola, IFEG, CONICET, \\ Ciudad Universitaria (5000) Córdoba, Argentina \\ (Received 18 June 2013; published 27 January 2014)
}

\begin{abstract}
A universal inequality that bounds the angular momentum of a body by the square of its size is presented and heuristic physical arguments are given to support it. We prove a version of this inequality, as consequence of the Einstein equations, for the case of rotating axially symmetric, constant density, bodies. Finally, the physical relevance of this result is discussed.
\end{abstract}

DOI: 10.1103/PhysRevLett.112.041101

PACS numbers: 04.70.Bw, 02.40.Ky, 04.20.Ex

Introduction.-Consider a rotating body $U$ with angular momentum $J(U)$. Let $\mathcal{R}(U)$ be a measure (with units of length) of the size of the body. A precise definition for the radius $\mathcal{R}$ will be given later on, for the present discussion it is enough to consider only the intuitive idea of size: for example, if the body is a sphere in flat space then $\mathcal{R}$ should be proportional to the radius of the sphere.

We conjecture that there exists a universal inequality for all bodies of the form

$$
\mathcal{R}^{2}(U) \gtrsim \frac{G}{c^{3}}|J(U)|,
$$

where $G$ is the gravitational constant and $c$ the speed of light. The symbol $\gtrsim$ is intended as an order of magnitude, the precise universal (i.e., independent of the body) constant will depend, of course, on the definition of $\mathcal{R}$.

The purpose of the first part of this Letter is to provide physical arguments supporting the validity of this inequality. In the second part we prove, as a consequence of the Einstein field equations, Theorem 1 . This theorem provides a precise version of the inequality (1) valid for rotating, axially symmetric, constant density, bodies. Finally, we conclude with a discussion of the physical relevance of this result.

Heuristic arguments. - The arguments in support of the inequality (1) are based in the following three physical principles: (i) the speed of light $c$ is the maximum speed; (ii) for bodies which are not contained in a black hole the following inequality holds:

$$
\mathcal{R}(U) \gtrsim \frac{G}{c^{2}} m(U),
$$

where $m(U)$ is the mass of the body; and (iii) the inequality (1) holds for black holes. Let us discuss these assumptions. Item (i) is clear. Item (ii) is called the trapped surface conjecture [1]. Essentially, it says that if the reverse inequality as in (2) holds then a trapped surface should enclose $U$. That is, if matter is enclosed in a sufficiently small region, then the system should collapse to a black hole. This is related to the hoop conjecture [2] (see also [3-5]). The trapped surface conjecture has been proved in spherical symmetry [6-8] and also for a relevant class of nonspherical initial data [9]. The general case remains open but it is expected that some version of this conjecture should hold.

Concerning item (iii), the inequality

$$
A \geq 8 \pi \frac{G}{c^{3}}|J|
$$

was recently proved for axially symmetric black holes (see [10] and references therein), where $A$ is the area of the stable marginally trapped surface and $J$ its angular momentum. The area $A$ is a measure of the size of a trapped surface, hence the inequality (3) represents a version of (1) for axially symmetric black holes. In fact the inequality (3) was the inspiration for the inequality (1). A possible generalization of (3) for bodies is to take the area $A(\partial U)$ of the boundary $\partial U$ of the body $U$ as measure of size. But unfortunately the area of the boundary is not a good measure of the size of a body in the presence of curvature. In particular, an inequality of the form $A(\partial U) \gtrsim$ $G c^{-3}|J(U)|$ does not hold for bodies. The counterexample is essentially given by a rotating torus in the weak field limit, with large major radius and small minor radius. The details of this calculation will be presented elsewhere [11].

It is important to emphasize that principles (i) and (iii) have a different status than principle (ii). The former are well-established facts; the latter is a conjecture. Assuming (i), (ii), and (iii) we want to argue that (1) should hold. Consider, in Newton theory, an axially symmetric body $U$ with mass density $\bar{\mu}$, rotating around the axis of symmetry with angular velocity $\omega$. These functions are not required to be constant on $U$. The angular momentum and the total mass of the body are given by

$$
J(U)=\int_{U} \bar{\mu} \omega \rho^{2} d v_{0}, \quad m(U)=\int_{U} \bar{\mu} d v_{0},
$$


where $\rho$ is the Euclidean distance to the axis and $d v_{0}$ is the Euclidean volume element. The angular velocity is bounded by

$$
|\omega|=\frac{|v|}{\rho} \leq \frac{c}{\rho},
$$

where we have used the principle (i): $|v| \leq c$, where $v$ is the linear velocity. Using (5) in the expression for the angular momentum (4) we obtain

$$
|J(U)| \leq c \int_{U} \bar{\mu} \rho d v_{0} \leq c m(U) \sup _{U} \rho .
$$

Note that this inequality is deduced using only the Newtonian expression for the angular momentum and principle (i). If the body is contained in a black hole, then the inequality (1) holds for the black hole boundary according to principle (iii). Hence, we assume that it is not contained in a black hole, and then, according to principle (ii), the inequality (2) holds. Using this inequality for the mass in (6) we get

$$
\frac{G}{c^{3}} J(U) \lesssim \mathcal{R}(U) \sup _{U} \rho .
$$

A reasonable property for a size measure (at least in flat space) is that

$$
\sup _{U} \rho \leq \mathcal{R}(U) .
$$

Using (8) in (7) we obtain (1). Note that even if the property (8) does not hold, the right-hand side of (7) can be interpreted as the square of a measure of the size of $U$ and hence an inequality of the form (1) also holds for that new measure of size.

It is clear that one of the main difficulties in the study of inequalities of the form (1) and (2) is the very definition of the quantities involved, in particular the measure of size. In fact, despite the intensive research on the subject, there is no known universal measure of size such that the trapped surface conjecture (or, more generally, the hoop conjecture) holds (see the interesting discussions in $[4,12,13]$ ). However, as we will see in the next section, the remarkable point is that in order to find an appropriate measure of size $\mathcal{R}$ such that (1) holds we do not to need to prove first (2), and hence we do not need to find the relevant measure of mass $m(U)$ for the trapped surface conjecture.

The arguments of the previous discussion can be summarized as follows. In order to increase the angular momentum of a body with fixed size there are two mechanisms: to increase the angular velocity or to increase the mass inside the body. But there is a physical limit to both mechanisms. The angular velocity is bounded by the speed of light, and increasing the mass (at fixed size) will eventually produce a black hole, where the inequality (1) holds. Hence, a universal inequality of the form (1) is expected for all bodies.

A precise version of the inequality.-We make precise the three notions involved in the inequality (1): a body $U$, the angular momentum $J$, and the size $\mathcal{R}$ of the body. A body $U$ is a connected open subset $U \subset S$ with smooth boundary $\partial U$; where $S$ is a spacelike three-surface which gives rise to the initial data set for Einstein equations defined as follows. An initial data set for the Einstein equations is given by $\left(S, h_{i j}, K_{i j}, \mu, j^{i}\right)$ where $S$ is a connected threedimensional manifold, $h_{i j}$ a (positive definite) Riemannian metric, $K_{i j}$ a symmetric tensor field, $j^{i}$ a vector field, and $\mu$ a scalar field on $S$, such that the constraint equations

$$
\begin{gathered}
D_{j} K^{i j}-D^{i} K=-8 \pi \frac{G}{c^{4}} j^{i}, \\
R-K_{i j} K^{i j}+K^{2}=16 \pi \frac{G}{c^{4}} \mu,
\end{gathered}
$$

are satisfied on $S$. Where $D$ and $R$ are the Levi-Civita connection and the scalar curvature associated with $h_{i j}$, and $K=K_{i j} h^{i j}$. In these equations the indices are moved with the metric $h_{i j}$ and its inverse $h^{i j}$. In terms of the fourdimensional energy momentum tensor $T_{\mu \nu}$, the matter fields are given by $\mu=T_{\mu \nu} n^{\mu} n^{\nu}, j_{\nu}=-h_{\nu}{ }^{\lambda} T_{\lambda \nu} n^{\nu}$, where $n^{\nu}$ is the timelike unit vector normal to the slice $S$. The relation between the mass density $\bar{\mu}$ used in (4) and the energy density $\mu$ is given by $\mu=c^{2} \bar{\mu}$.

We require that the matter fields satisfy the dominant energy condition

$$
\mu \geq \sqrt{j^{i} j_{i}} .
$$

In order to have a proper definition of the angular momentum of the body we will further assume that the data are axially symmetric (in general, the angular momentum of a bounded region $U$ is very difficult to define; see the review article [14] and reference therein). That is, we assume the existence of a Killing vector field $\eta^{i}$, i.e.,

$$
\mathcal{L}_{\eta} h_{i j}=0,
$$

where $\mathcal{L}$ denotes the Lie derivative, which has complete periodic orbits and such that

$$
\mathcal{L}_{\eta} \mu=\mathcal{L}_{\eta} j^{j}=\mathcal{L}_{\eta} K_{i j}=0 .
$$

We denote the norm of the Killing vector by $\lambda=\left(\eta^{i} \eta_{i}\right)^{1 / 2}$. The angular momentum of the body $U$ is defined by

$$
J(U)=-\frac{1}{c} \int_{U} j_{i} \eta^{i} d v
$$

where $d v$ is the volume measure with respect to the metric $h_{i j}$. 
Finally, we should define a notion of size for the body $U$. This notion will be a variant of the following definition of radius presented by Schoen and Yau in [15]. Let $\Gamma$ be a simple closed curve in $U$ which bounds a disk in $U$. Let $p$ be largest constant such that the set of points within a distance $p$ of $\Gamma$ is contained within $U$ and forms a proper torus. Then $p$ is a measure of the size of $U$ with respect to the curve $\Gamma$. The radius $\mathcal{R}_{\mathrm{SY}}(U)$ is defined as the largest value of $p$ we can find by considering all curves $\Gamma$. That is, $\mathcal{R}_{\mathrm{SY}}(U)$ is expressed in terms of the largest torus that can be embedded in $U$. Using this definition, the following deep theorem was proved in [15]. Let $U$ be any subset of $S$. Assume that the scalar curvature $R$ of the metric $h_{i j}$ is bounded from below $R \geq \Lambda$ in $U$ by a positive constant $\Lambda$. Then the following inequality holds:

$$
\Lambda \leq \frac{8 \pi^{2}}{3} \frac{1}{\mathcal{R}_{\mathrm{SY}}^{2}} .
$$

Note that this is a purely local and purely Riemannian result. There is no requirement that $S$ be asymptotically flat and only assumptions on the metric $h_{i j}$ are made.

In [16] O'Murchadha made the following important observation. Define another radius $\mathcal{R}_{O M}(U)$ as follows. Let $\mathcal{R}_{O M}(U)$ be the size of the largest stable minimal twosurface that can be imbedded in $U$, where size of the surface is the distance (with respect to the ambient metric $h_{i j}$ ) from the boundary to that internal point which is furthest from the boundary. Then, it can be proved that

$$
\mathcal{R}_{O M}(U) \geq \mathcal{R}_{\mathrm{SY}}(U),
$$

and also that the same bound (15) holds for $\mathcal{R}_{O M}(U)$, under similar assumptions [17]. Namely,

$$
\Lambda \leq \frac{8 \pi^{2}}{3} \frac{1}{\mathcal{R}_{O M}^{2}} .
$$

Since we have (16), the right-hand side of (17) is smaller than the right-hand side of (15), and hence $\mathcal{R}_{O M}$ provides a better bound.

To have an intuitive idea of these measures, let us compute them for some relevant domains in flat space. Recall that the planes are minimal stable surfaces in flat space. For a sphere of radius $b$ we have that $\mathcal{R}_{\mathrm{SY}}=b / 2$, $\mathcal{R}_{O M}=b$. We see that both radii give essentially the same desired value for the sphere. For a torus with major radius $b$ and minor radius $a$ we have $\mathcal{R}_{\mathrm{SY}}=a / 2, \mathcal{R}_{O M}=a$. Both radii are independent of the major radius $b$ for the torus. Hence, we cannot expect an inequality of the form (1) for $\mathcal{R}_{\mathrm{SY}}$ or $\mathcal{R}_{O M}$, since in the weak field limit a torus of large radius $b$ and small radius $a$ will have large angular momentum $J$ and small $\mathcal{R}_{\mathrm{SY}}$ or $\mathcal{R}_{O M}$ (a similar counterexample as in the case of the area discussed above). Finally, to see the relevant difference between $\mathcal{R}_{\mathrm{SY}}$ and $\mathcal{R}_{O M}$ consider a cylinder with radius $a$ and height $L$. We have $\mathcal{R}_{\mathrm{SY}}=\min \{a / 2, L / 2\}, \mathcal{R}_{O M}=a$. When $L>a$, then both radii give similar values, however for a thin disk with $L<a$ we have $\mathcal{R}_{\mathrm{SY}}=L / 2$ and $\mathcal{R}_{O M}=a$. That is, $\mathcal{R}_{\mathrm{SY}} \rightarrow 0$ as $L \rightarrow 0$ while $\mathcal{R}_{O M}$ is independent of $L$.

Motivated by the example of the torus, we define a new radius for axially symmetric bodies as follows. Considering a region $U$ with a Killing vector $\eta^{i}$ with norm $\lambda$, we define the radius $\mathcal{R}$ by

$$
\mathcal{R}(U)=\frac{2}{\pi} \frac{\left(\int_{U} \lambda d v\right)^{1 / 2}}{\mathcal{R}_{O M}(U)} .
$$

This will be our measure for size for the inequality (1). The most natural normalization for $\mathcal{R}$ in the inequality (1) is to require that $\mathcal{R}=b$ for an sphere in flat space of radius $b$. This is the reason for the factor $2 / \pi$ in (18).

We have also the analog definition with respect to $\mathcal{R}_{\mathrm{SY}}$, namely

$$
\mathcal{R}^{\prime}(U)=\frac{2}{\pi} \frac{\left(\int_{U} \lambda d v\right)^{1 / 2}}{\mathcal{R}_{\mathrm{SY}}(U)} .
$$

Using the inequality (16), we obtain

$$
\mathcal{R}^{\prime}(U) \geq \mathcal{R}(U) .
$$

That is, from the point of view of the inequality (1), the radius $\mathcal{R}$ provides a sharper estimate than $\mathcal{R}^{\prime}$.

For the torus in flat space, the volume integral of the norm of the Killing vector is given by

$$
\int_{\text {Torus }} \rho d v_{0}=2 \pi^{2} a^{2}\left(\frac{a^{2}}{4}+b^{2}\right) .
$$

Then we obtain

$$
\mathcal{R}=2^{3 / 2}\left(\frac{a^{2}}{4}+b^{2}\right)^{1 / 2}, \quad \mathcal{R}^{\prime}=2 \mathcal{R} .
$$

The important point is that in the limit $a \rightarrow 0$ we obtain $\mathcal{R}=2^{3 / 2} b$, that is, a torus with a large $b$ has also large size in contrast with the original radii $\mathcal{R}_{\mathrm{SY}}$ or $\mathcal{R}_{O M}$. For a thin disk with $L<a$ we have

$$
\mathcal{R}=\frac{2^{3 / 2}}{\sqrt{3 \pi}} \sqrt{a L}, \quad \mathcal{R}^{\prime}=\frac{2^{5 / 2}}{\sqrt{3 \pi}} \frac{a^{3 / 2}}{L^{1 / 2}} .
$$

We see that $\mathcal{R} \rightarrow 0$ and $\mathcal{R}^{\prime} \rightarrow \infty$ as $L \rightarrow 0$. That is, the difference between the two measures is significant.

Finally, it is important to compute $\mathcal{R}$ for a very dense body where the gravitational field is strong. Consider a constant density star of total mass $m$ with area radius equal to Schwarzschild radius $2 m G / c^{2}$. That is, we are considering the limit case before the formation of a black hole. 
The radius $\mathcal{R}_{O M}$ for that case was calculated in [16]. Using that result we obtain

$$
\mathcal{R}=\frac{2^{11 / 2}}{\pi \sqrt{3}} \frac{G}{c^{2}} m \approx 8.16 \frac{G}{c^{2}} m .
$$

We see that $\mathcal{R}$ is of the same order of magnitude than the area radius, and hence it is a reasonable measure of size in that case.

We have the following result.

Theorem 1 Let $\left(S, h_{i j}, K_{i j}, \mu, j^{i}\right)$ be an initial data set that satisfy the energy condition (11). We assume that the data are maximal (i.e., $K=0$ ) and axially symmetric. Let $U$ be an open set in $S$. Assume that the energy density $\mu$ is constant on $U$. Then the following inequality holds:

$$
\mathcal{R}^{\prime 2}(U) \geq \frac{24}{\pi^{3}} \frac{G}{c^{3}}|J(U)| .
$$

The same bound holds for $\mathcal{R}(U)$ if we assume, in addition, that the boundary $\partial U$ is mean convex.

Proof. The angular momentum of the body $U$ is given by (14). Define the unit vector $\hat{\eta}^{i}$ by

$$
\hat{\eta}^{i}=\frac{\eta^{i}}{\lambda} .
$$

Then we have

$$
\begin{aligned}
|J(U)| \leq & \frac{1}{c} \int_{U}\left|j^{i} \eta_{i}\right| d v=\frac{1}{c} \int_{U}\left|j^{i} \hat{\eta}_{i}\right| \lambda d v \\
& \leq \frac{1}{c} \int_{U} \sqrt{j^{i} j_{i}} \lambda d v \\
& \leq \frac{1}{c} \int_{U} \mu \lambda d v,
\end{aligned}
$$

where in the line (28) we have used that $\hat{\eta}^{i}$ has unit norm, in the line (29) we used the energy condition (11).

We have assumed that the data are maximal and hence, by equation (10), we obtain

$$
R \geq 16 \pi \frac{G}{c^{4}} \mu .
$$

Since we have assumed that $\mu$ is constant [which should be positive by the energy condition (11)] on $U$, we can take $\Lambda=16 \pi G c^{-4} \mu$ and then we are under the hypothesis of the Schoen-Yau theorem. That is, the bound (15) holds, and hence we get

$$
\mu \leq \frac{\pi}{6} \frac{c^{4}}{G} \frac{1}{\mathcal{R}_{\mathrm{SY}}^{2}} .
$$

Using this bound in (29) we obtain

$$
|J(U)| \leq \frac{\pi}{6} \frac{c^{3}}{G} \frac{1}{\mathcal{R}_{O M}{ }^{2}} \int_{U} \lambda d v=\frac{\pi^{3}}{24} \frac{c^{3}}{G} \mathcal{R}^{\prime 2},
$$

where in the last equality we have used the definition (19). Under the additional assumption that the boundary $\partial U$ is mean convex, we have the same bound (31) for the radius $\mathcal{R}_{O M}$, and hence the same inequality (32) holds for $\mathcal{R}$.

It is interesting to note that this proof is very similar to the heuristic argument presented above. There is a physical reason for this similarity: in axial symmetry the gravitational waves have no angular momentum. All the angular momentum is contained in the matter sources. Hence the Newtonian expression for the angular momentum (4) is similar to relativistic one (14). Condition (i) on the maximum velocity of the matter is expressed in the dominant energy condition (11). Moreover, from inequality (29) (without using the assumption that $\mu$ is constant), we get the analog of the inequality (6), namely

$$
|J(U)| \leq \operatorname{cm}(U) \sup _{U} \lambda,
$$

where we have defined

$$
m(U)=\frac{1}{c^{2}} \int_{U} \mu d v .
$$

Note that the length of the azimuthal circles is given by $2 \pi \lambda$, hence $\lambda$ represents a natural generalization for curved spaces of the coordinate $\rho$ that appears in (6).

The important new ingredient is that instead of using the bound (2) for the mass of the body, we use the Schoen-Yau bound for the energy density (15). This allows us to bypass the hoop conjecture and its associated definition of size and mass.

Note that the radius used in the theorem cannot be applied in general to black holes, since it requires a regular interior region. And even when the interior is regular the radius is not a priori related to the black hole area. A relevant open problem is to find a suitable measure of size that can be applied for both black holes and bodies.

Physical relevance. - It is important to emphasize that the validity of inequality (1) is entirely independent of any specific matter model; the only requirement is that the dominant energy condition is satisfied.

The inequality (1) is a prediction of Einstein theory and hence it should be contrasted with observational evidences. In order to violate this inequality a body should be small and highly spinning, a natural candidate for that is a neutron star. For the fastest rotating neutron star found to date (see [19]) we have

$$
\omega \approx 4.5 \times 10^{3} \mathrm{rad} \mathrm{s}^{-1} .
$$


Assuming that the neutron star has about three solar masses (which appears to be a reasonable upper bound for the mass; see [20]) we obtain

$$
m \omega \approx 2.7 \times 10^{37} \mathrm{~s}^{-1} \mathrm{~g} .
$$

The radius of the neutron star is typically

$$
\mathcal{R} \approx 1.2 \times 10^{6} \mathrm{~cm} .
$$

Assuming that the star is spherical with constant density we get that the angular momentum is given by

$$
\frac{G}{c^{3}}|J|=\frac{G}{c^{3}} \frac{2}{5} m \mathcal{R}^{2} \omega \approx 3.8 \times 10^{10} \mathrm{~cm}^{2} .
$$

This should be compared with the square of the radius

$$
\mathcal{R}^{2} \approx 1.44 \times 10^{12} \mathrm{~cm}^{2} .
$$

We see that the inequality (1) is satisfied.

Finally, it is also interesting to consider what kind of limit the inequality (1) imposes on elementary particles. From quantum mechanics we get that the angular momentum of an elementary particle is given by

$$
J=\sqrt{s(s+1)} \hbar, \quad \hbar=1.05 \times 10^{-27} \mathrm{~cm}^{2} \mathrm{~s}^{-1} \mathrm{~g},
$$

where $s$ is the spin of the particle. Using this expression in (1) we obtain that the classical theory impose the following minimal size for a particle with spin $s$ :

$$
\mathcal{R}_{0}=[s(s+1)]^{1 / 4} l_{p}, \quad l_{p}=\left(\frac{G \hbar}{c^{3}}\right)^{1 / 2},
$$

where $l_{p}=1.6 \times 10^{-33} \mathrm{~cm}$ is the Planck length. We recover the Planck length essentially because the order of magnitude of the universal constant in the inequality (1) is one. It appears to be a remarkable self-consistence of the Einstein field equations that they predict a minimum length of the order of magnitude of the Planck length if we assume that there exists a minimum for the angular momentum given by quantum mechanics.
It is a pleasure to thank E. Gallo, G. Galloway, R. J. Gleiser, N. Ó Murchadha, O. Ortiz, M. Reiris, R. Wald, for illuminating discussions. This work was supported by Grant No. PICT-2010-1387 of CONICET (Argentina) and Grant No. Secyt-UNC (Argentina).

*dain@famaf.unc.edu.ar

[1] H. Seifert, Gen. Relativ. Gravit. 10, 1065 (1979).

[2] K. Thorne. Nonspherical gravitational collapse: A short review. In Magic Without Magic: John Archibald Wheeler. A Collection of Essays in Honor of his Sixtieth Birthday, edited by J. Klauder (W.H. Freeman, San Francisco, 1972), pp. 231-258.

[3] E. Flanagan, Phys. Rev. D 44, 2409 (1991).

[4] E. Malec, Acta Phys. Pol. B 22, 829 (1992).

[5] R. Wald. Gravitational collapse and cosmic censorship. In Black Holes, Gravitational Radiation and the Universe, edited by B. R. Iyer and B. Bhawal, Fundamental Theories of Physics Vol. 100 (Kluwer Academic, Dorddrecht, 1999), pp. 69-85.

[6] P. Bizon, E. Malec, and N. O’Murchadha, Phys. Rev. Lett. 61, 1147 (1988).

[7] P. Bizon, E. Malec, and N. O'Murchadha, Classical Quantum Gravity 6, 961 (1989).

[8] M. A. Khuri, Phys. Rev. D 80, 124025 (2009).

[9] E. Malec, Phys. Rev. Lett. 67, 949 (1991).

[10] S. Dain, Classical Quantum Gravity 29, 073001 (2012).

[11] P. Anglada, S. Dain, and O. Ortiz (unpublished).

[12] G. Gibbons, AIP Conf. Proc. 1460, 90 (2012).

[13] J. M. Senovilla, Europhys. Lett. 81, 20004 (2008).

[14] L. B. Szabados, Living Rev. Relativity 7, 4 (2004).

[15] R. Schoen and S. T. Yau, Commun. Math. Phys. 90, 575 (1983).

[16] N. O’Murchadha, Phys. Rev. Lett. 57, 2466 (1986).

[17] It was pointed out in [18] that to prove this bound with the radius $\mathcal{R}_{O M}$ an additional requirement is needed: the boundary $\partial U$ should be mean convex.

[18] G. J. Galloway and N. O'Murchadha, Classical Quantum Gravity 25, 105009 (2008).

[19] J. W. Hessels, S. M. Ransom, I. H. Stairs, P. C. C. Freire, V. M. Kaspil, F. Camilo, Science 311, 1901 (2006).

[20] J. Lattimer and M. Prakash, Science 304, 536 (2004). 